\title{
Emotion Recognition of Modern Graphic Design Language
}

\author{
Zhaohui Yuan \\ Academy of Fine Arts \\ Huanggang Normal College \\ Huanggang, China
}

\begin{abstract}
Emotion is the attitude experience of graphic language composition and expression. In this paper, the emotional thinking in graphic design is decomposed into three levels: expression signal, emotional experience and emotional infiltration, the relationship between emotion and graphic image, shape and image is discussed, and the characteristics and methods of emotional recognition in modern graphic design language is expatiated.
\end{abstract}

Keywords—graphic language; expression; mood; emotion

\section{INTRODUCTION}

"Graphics is a world language that transcends the barriers between counties and nations. Its commensuration makes up the boundaries between nations and regions and fills the gap between the masses." [1] When we contact a graphic, its modeling element and art form will form a whole impression in our mind, Mr. Yin Dingbang said graphics' "commensuration" refers not only the form of graphical information exchange, but also should include the transformed general attitude when the audience interpret these graphics, which is a psychological impact under the emotional reflection. Graphic as a kind of "emotional" visual symbol, in ancient times recorded the emotional activities during human survival and reproduction, and along with the continuous expansion of human cognitive experience and subjective experience, the graphical language with emotional consensus gradually formed into a unique visual language system which is used to convey information and express emotional world.

Human emotions are complex and rich, emotional recognition needs to be studied from various perspectives. As a psychological concept, emotion first is the attitude of psychological response; from the physiological point of view, emotion is a mood reflection which generated by visual stimulation, accompanied by feelings; at last from the humanity point of view, emotion is desire and expectation, it is the feelings of the inner world of people. In modern graphic design, emotional activities always run through it, graphic design language in a sense is the emotional coding method of visual elements and thinking activities. Emotion not only is a part of the graphic information content, but also the catalyst of graphic meaning interpretation, designer through the "emotional expression" to achieve the effective transmission of graphical information, and the audience through a certain "emotional recognition" approach to grasp the spirit and meaning of graphics.

\section{THE EXPRESSION SIGNAL OF IMAGE}

Expression is the psychological attitude that first is aware in interpersonal communication. Psychologists study the human facial muscle movements, and then set up interest, pleasure, surprise, sadness, anger, disgust, fear, pain, contempt and other basic emotions. Human expressions is not only presented through facial manifestations, but also presented through the action characteristics of gesture and language rhythm. From modern communication theory, expression is the "non-language information dissemination system" in interpersonal communication.

In original graphic art, the mankind expresses their thoughts and feelings through facial expressions, such as the painted pottery basin with human face and fish pattern, based on human face, assembled fish, birds, net pattern together into decorative graphics, to symbolize the beautiful prayer for the richness of life. In modern society, people use human face and action gesture to design and produce emotion icons, which has been widely used in network and communication exchanges. Americans combine emotion and icon into a new vocabulary expression symbol "Emoticon", called "Yan text" by Japanese, referring to the writer's mood. When Emotional symbols convey information, they could be both fast, intuitive, and also they could more subtly and implicitly express emotional attitudes and psychological reactions, such as when the mood is bad, there are surprise, tiredness, grievances, sadness, injury, tears, cry, rage and other emoticons. It can be said that expression in interpersonal communication is the first thing to be aware and understood, so it not only could go beyond the racial and language barriers, but also could express the delicate and rich psychological state, playing a catalytic role for information exchange.

Psychologists believe that expression hints are more effective than language. During the two world wars, the war posters became important political propaganda tools for governments, which are mostly about soldier recruitment, patriotic propaganda, front-line support, rear defence and so on. In the Soviet war themed poster "March! Victory Is in Hand!", the main image of the graphics is a portrait of Soviet Red Army head, whose fortitude facial expression and firm eyes bring people inspiring feelings, and the graphics in the first 
time conveys the strategic situation of victory in hand and inspiring expression signal. Modern graphic design not only could use facial expressions, but also could use the gesture and action characteristics of human or animal images to release the expression signal. In Mr. Jin Taiqiang's silverware advertising "Hand in Hand", the graphics do not use intuitive facial expressions, but the delicate touch characteristics of mother and child's hands to convey a happy expression.

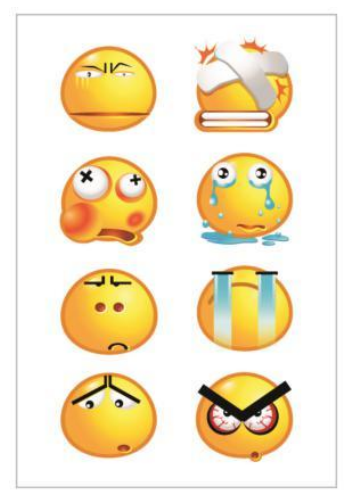

Fig. 1. Network emotion.

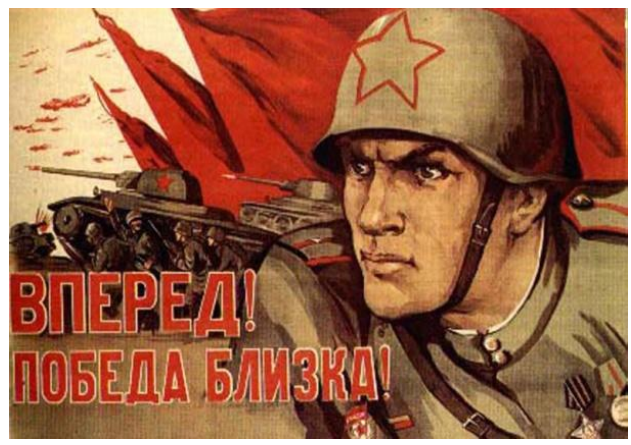

Fig. 2. The war poster of the Soviet Union Go ahead! Win and win.

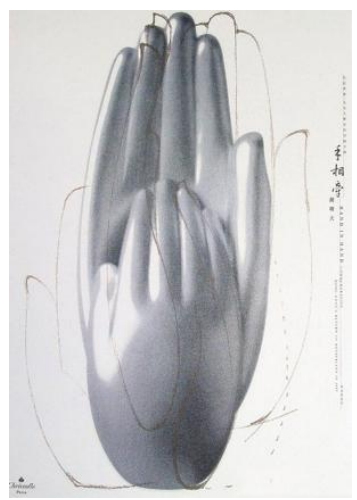

Fig. 3. 图 3 The silverware advertisement of hands in hands.

\section{THE EMOTIONAL EXPERIENCE OF GRAPHICAL FORM}

The graphic itself may be just a combination of visual image or multiple images. These reorganized, reconstructed forms stimulate the audience's cognitive graphics, also affect the psychological feelings of human beings to generate visual emotions. Visual emotions not only could be formed through association and imagination, and along with the individual experience become richer. "People's emotions are often influenced by many factors such as age, experience, personality, environment and so on. Visual emotions are also closely related to these factors, and influenced by individual experience and hobby, quality and choice such factors." [2] Who do not have certain knowledge of visual arts, often stay in the outside images which can stimulate interest, but after the systematic visual art training, they will be able to look into the formal structure and visual element which are implied in graphic shapes.

Graphic shape contains two meanings: "shape" is the basic contour of visual object, the image features of shape constitute a basic understanding for various graphics, the image association leads to the theme-associated concept; and "form" refers to the internal structure relation between visual "force style" and the combination of graphic elements, which has cultural and spiritual characteristics. The basic elements of graphic form are point, line, surface and their combination forms, which constitute the medium that the emotions depend on. These elements have no specific meaning of themselves, they depend on size, direction, severity, light and shade, density, texture, rhythm to form the abstract spiritual symbol. Susan Lange from the perspective of semiotics, believed that people during the process of understanding things, there is a "perceptive shape language" and "logical text language", "Artwork is the projection of emotional life in space, time or poem, so that artwork is also a form of emotion." [3]. As a perceptual thinking mode, emotion in visual psychology is mainly involved in the perception and experience of the form, therefore, "looking" at the graphical form will have a subjective feeling of emotional experience. In graphic design, emotional experience is often generated on the base of the emotional stimulation, with an induced form and language structure to form the complex emotional semantics which relates to people's social needs.

In the poster "Merry Christmas", the graphics body is a Christmas tree which is collaged by the broken world map, the shape of the Christmas tree in design is intentionally divided from structure, and the rectangular stitching is used for graphics bottom. The war, conflict, poaching, whaling, refugees in the rectangular picture of bottom formed a formal unity with the main graphics, and in the overall structure it shows the fragmentary patching and forced integration, and the fuzzy form and incompleteness expresses the question to the joy of Christmas and global carnival. The author borrows the postmodern metaphorical language and double decoding as the cultural symbol of Western festivals, with the merry Christmas and global carnival established based on endless unbridled plunder of resources, showing a helpless mood after foreign cultural aggression.

In graphic design language, through the reconstruction of the relational model between shape and meaning, it is often composed of multiple meanings and expression semantics to form the semantic meaning, and integrates specific culture, concept and style with certain rhetorical devices into it, in order to constitute the emotion appeal with complete graphic form. If the human visual experience is a kind of image cognition, emotional experience is emphasis on the form 
"empathy" and "poetic" understanding. Along with the upgrade of visual form from experience to taste and the gradual upgrade of the graphics understanding level, the socalled "meaningful form" is the stylized "translation" of graphic meaning under emotional experience.
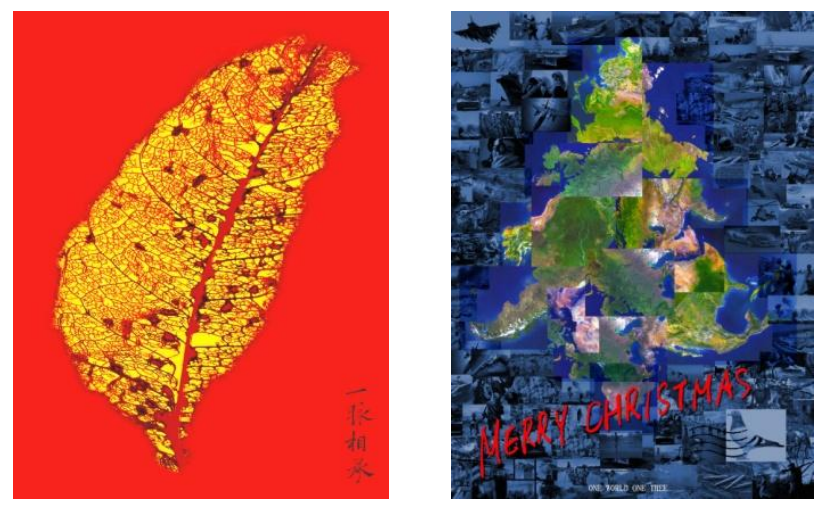

Fig. 4. posters of Merry Christmas and the same strain.

\section{THE EMOTIONAL PENETRATION OF GRAPHIC IMAGES}

"The most heart touching is emotional feeling", the feelings are at the deepest subconscious place of emotional activity; artistic feelings and feelings of life are different, which is a filtered and extracted aesthetic feeling, Feuerbach believes that feeling can only dialogize with feelings but the charm of the graphics lies in the power of emotion, and when the graphics makes dialogue to the audience with feelings, the graphics language finally impresses people's mind with image rendering.

The image is a simulacrum of objective object in people's inner heart, which has a strong emotional color, and it is the objective "image" that is brought by the "meaning" of subjective feelings, and is an overall feeling rendering to graphics. Gestalt theory is to grasp and construct external image from an "overall relationship", and in this sense, image is a structural system, and people's inner heart driven by the subjective feelings can take the initiative to process the objective image. Parker elaborated the aesthetic experience of art from an intuitive and introspective way, he believed that "the purpose of art is to show a specific soul state with certain emotion as the core ... especially the whole object is necessary, because our feelings rely on the whole."[4] In a sense, the graphics image is to cause a deep sense of infiltration through a form of overall mood, and the interpretation of the image is to explain the overall abstract spirit of graphic form.

In the poster "The Same Strain", the first visual impression of the graphic is a leaf; the relationship between outline and background is simple; leaf shape is tilted with a destabilizing factor, which makes the graphics show a strong visual impact and tension, thus to constitute the graphic expression signal of self-emphasizing and appeal. The leaf as a creative element has the similar outline with Taiwan map, with an image similarity association, which is also the creative basis of graphical elements isomorphism. In the thinking habits of local culture, we will associate leaf with tree, to further extend the concept through the association and imagination; leaf and
Taiwan, tree and the mainland constitute a symbolic relationship. During the further processing of visual texture in graphics forms, it changes the leaf vein texture into vascular vein, symbolizing Taiwan and the mainland have the same strain, the same culture and the same cultural identity. The isomorphism of leaf veins, vascular vein and cultural vein touches a poetic mood of "blood being thicker than water", and then triggers audiences' imagination and expectation of "child returning back to mother". In color use, red and yellow metaphor that they are all refer to the descendants of the Yan and Huang Emperors, black color in the texture intensifies the visual contrast to produce tension mood. Together with the tension mood from the image experience eventually evolved complex, delicate and rich feelings. Graphics chooses leaf image that has the cultural and emotional consensus as the metaphor, integrates the subjective passion and objective form, closely linked to human mind, arouses a strong national unity willing and eventually reaches the dialogue of graphics and audience to produce emotional resonance.

It can be seen that Graphic image organically integrates the "meaning" and "image" of graphical form, and more importantly the image infection makes the emotional resonance happen between audience and graphics, further sublimate into a subjective will and spiritual pursuit. The image appearance and the rendering of graphic language are an organic whole. The designer in graphic design language expression, guides the graphics creation with real feelings, changes the forms derived from the inner into a graphic language with spirit and meanings.

\section{CONCLUSION}

The rise of modern linguistics, psychology, communication, semiotics, digital media technology and other fields have provided interdisciplinary multi-perspective for graphic design. As a visual language, the value of graphics not only stays in the function of information transmission, but also affects the aesthetic standards and behavior of an era, and then plays a role of communication and cultural exchange. From the perspective of the origin and development of art and design, any doctrine can not replace the life-oriented sentiment and emotional experience, excellent designers not only need to be sensitively concerned about society and life, enrich design inspiration, but also need to sensitively grasp audiences' "emotional needs", develop graphics language field under the real emotional guidance, so that the emotional recognition of graphic design language has a stronger pertinence, effectiveness and integrity.

\section{REFERENCES}

[1] Yin Dingbang: Graphics and significance [M], Hunan Science and Technology Press, Changsha, 2003, p.4

[2] Wu Lieyan: Visual experience [M], Jiangsu Fine Arts Publishing Company, Nanjing, 2008, p. 9.

[3] [US]Susan Lange: Emotion and Form [M],Translated by Liu Daji, Fu Zhiqiang, Zhou Faoxiang, China Science and Society Press, Beijing, 1986, p. 24

[4] [US] H.Parker: Aesthetic Principle [M], Translated by Zhang Jin , Guangxi Normal University Press, Guilin, 2001, p. 216. 